\title{
Articles
}

\section{The Assessment of Carbendazim, Cyazofamid, Diethofencarb and Pyrimethanil Residue Levels in $P$. ginseng (C. A. Meyer) by HPLC}

\author{
Jeong-Heui Choi, ${ }^{\dagger \star \star}$ A. M. Abd El-Aty, ${ }^{\dagger, \star \star}$ Young-Seok Park, Soon-Kil Cho, and Jae-Han Shim ${ }^{\dagger *}$ \\ Natural Products Chemistry Laboraton, Institute of Agricultural Science and Technologv. Chomam National Lniversity. \\ Gwangin 500-757. Korea. ${ }^{*}$ E-mail jhshimächonnam ack \\ -Department of Pharmacologv, Facult of T'eterinary Medicine. Cairo Universitv. I2211-Giza, Egvpt \\ and Konkuk Lhiversit, Seoul 143-701. Korea

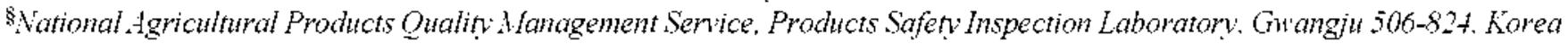 \\ Received November 21, 2006
}

\begin{abstract}
A fast and simple high-performance liquid chromatography (HPLC) method for the simultaneous determination of four pesticides having fungicide properties has been proposed for Panax ginseng, C. A. Meyer grown for 4 . 5. or 6 years. Analytical separation was performed on $\mathrm{C}_{18}$ columns using ultraviolet detector under gradient conditions. Spiked blank samples were used as standards to counteract the matrix effect observed in the chromatographic determination. The HPLC response for all pesticides was linear. with determination coefficients $>0.9986$. The average rate of recovery for pesticides spiked with 2 fortification levels was $>72 \%$ with relative standard deviations $<9 \%$. The limits of quantification (LOQ) ranged from 0.03 to $0.16 \mathrm{ppm}$. These LOQs were lower than the respective maximum residue limits (MRL) established by the Korean Food and Drug Administration (KFDA), except for cyazofamid. The proposed method was used to determine pesticide residue levels in samples of ginseng obtained from Jeonnam Province (Republic of Korea). None of the pesticides were found in ginseng samples grown for 4,5 , or 6 years
\end{abstract}

Key Words : Panar ginseng. Pesticides. Liquid chromatography

\section{Introduction}

Ponco ginseng (Asian ginseng). P. psendo-ginseng (Japanese ginseng) and $P$. quinquefolints (North American ginseng) represent primary sources of the herb that is commonly referred to as ginseng. The roots of $P$. ginseng. C.A. Meyrer. which has been used in traditional herbal remedies and medicines in Eastern Asia for more than 2000 years. are often used to make a tonic to increase energy: reduce susceptibility to various disorders and for recovery from illness. ${ }^{1.2}$ Ginseng is composed of a mixture of glycosides. essential oils. and a variety of complex carbohydrates and phytosterols. as well as amino acids and trace minerals. ${ }^{3}$ The principle active ingredient in ginseng (responsible for the biological effects) is believed to be a complex mixture of more than 30 triterpenoid saponins (each with a different set of properties) that are commonly referred to as ginsenosides and are present in the leaf. stem. and berries of the plant. in addition to their traditional source the root. ${ }^{+}$The pharmacological effects of ginseng include enhancement of carbohy'drate and lipid intermediary metabolism. ${ }^{5}$ and anti-stress mechanisms. ${ }^{6}$ There are also reports of antioxidant ${ }^{7,8}$ and anti-tumor activity for ginseng. ${ }^{9}$

Ginseng is highly susceptible to fungal diseases such as anthracnose $(20 \%-47 \%)$. damping-off $(5 \%-50 \%)$. root rot

${ }^{a}$ This paper is equally contributed by the two authors.
$(1 \%-60 \%)$. and alternaria blight $(10 \%-20 \%){ }^{10}$ This susceptibility is associated with its requirement for artificial shade and the fact that these plants have to grow for 4 to 6 years before they can be harvested. Pesticides are essential to control these diseases and increase harvest productivity: however. because of their potentially dangerous effects on human health. the control of pesticide residue in food is of great importance. The simultaneous presence of pesticides (organochlorine. carbamate and pyrethroid) in $P$. ginseng has rarely been considered in multiresidue analy ses. ${ }^{1.113}$

Organophosphorus pesticides have also been studied, because some of them are used extensively. ${ }^{12}$ In our previous study. we used gas cluromatography (GC) as a separation technique to analyze the pesticide residues in $P$. ginseng. Because of its high separation power and the availability of a variety of sensitive and selective detectors (e.g.. the electron capture detector and nitrogen-phosphorus detector). 18 pesticides (among 32 pesticides registered for ginseng in Republic of Korea) were monitored in ginseng matrices. ${ }^{13}$ The pesticide tolclofos-m has been detected in ginseng obtained from Jeonnam Province in the Republic of Korea ${ }^{13}$ In the present contribution, we measured the residue levels for 4 pesticides (carbendazim. cyazofamid. diethofencarb and pyrimethanil) that had been used in P. ginseng, based on liquid partitioning with an organic solvent (acetonitrile), purification by solidphase extraction. and high performance liquid chromatography. This method was applied to the determination of 
pesticides in ginseng samples obtained from the southern region of the Korean peninsula. It should be noted that the remaining compounds including metam-sodium. mancozeb. copper sulfate polyoxin B D zinc salt iminoctadine triacetate. metaldehyde. fludioxonil. chlorothalonil. and carbosulfan. could not be simultaneously detected by GC or HPLC.

\section{Experimental Section}

Materials. HPLC-grade acetonitrile. n-hexane. dichloromethane. methanol and anhydrous sodium sulphate were supplied by Merck (Darmstadt. Germany). The pesticides carbendazim (96.5\%), cyazofamid $(97.0 \%)$. diethofencarb $(98.5 \%)$, and pyrimethanil $(98.5 \%)$ were obtained from $\mathrm{Dr}$. Elurenstorfer (Augsburg. Germany): the chemical structures of these compounds are illustrated in Table 1. Water was distilled and filtered through a Milli-Q apparatus (Millipore. Seoul. Korea) before use. Stock pesticide solutions (1000 ppm) were prepared by dissolving each pesticide standard in methanol and storing at $4{ }^{\circ} \mathrm{C}$ in a brown bottle with a Teflonlined screw cup. Pesticide working-strength solutions were prepared by diluting the stock solutions in methanol. No degradation of the pesticides was detected under the storage conditions used at any time during the study.

Samples. Twelve samples (weight approx. $2 \mathrm{~kg}$ ) of ginseng roots harvested 4.5 , or 6 years after planting were

Table 1. Chemical structures, uses, and the retention times for the tested pesticides

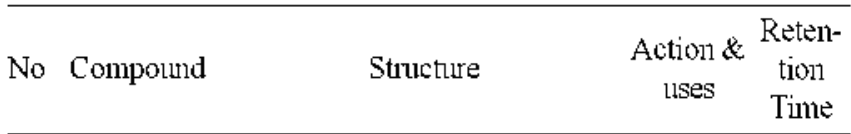

I carbendazim cyazotamid

Table 2. Conditions of HPLC analysis of pesticides in Panax ginseng (C. A. Meyer)

\begin{tabular}{|c|c|}
\hline Instrument & $\begin{array}{l}\text { HF-1100 Series (Agilent Technologies. Inc., } \\
\text { Palo Alto, Cálif, USA) }\end{array}$ \\
\hline Column & $\begin{array}{l}\text { Luna } C_{18}(25 \mathrm{~cm} \text {, particle size } 5 / \mathrm{mm} \text {, } \\
\text { Hhenomenex Hroducts, USA })\end{array}$ \\
\hline Detector & $\begin{array}{l}\text { UVD } \\
\text { Wátelength: } 254 \mathrm{~nm}\end{array}$ \\
\hline Injection volume & $10, t \mathrm{~L}$ \\
\hline Mobile phase & $\begin{array}{l}\mathrm{H}_{2} \mathrm{O}: \mathrm{CH}_{3} \mathrm{CN}=70.30,1.0 \mathrm{~mL} / \mathrm{min} \\
\text { (from } 0 \text { to } 5.0 \mathrm{~min} \text { ) } \\
\mathrm{H}_{2} \mathrm{O}: \mathrm{CH}_{3} \mathrm{CN}=15: 85,1.0 \mathrm{~mL} / \mathrm{min} \\
\text { (from } 5.0 \text { to } 50.0 \mathrm{~min} \text { ) }\end{array}$ \\
\hline
\end{tabular}

collected between April and October 2004 from 4 agricultural areas. Haenam. Naju. Yeongam and Yeonggwang in Jeonnam Province in the southern region of the Korean peninsula. Prior to the analysis. each sample was blended and divided into 4 parts. A $20-\mathrm{g}$ sample was selected randomly and tested in triplicate for pesticide residue levels using HPLC with a-UV detector.

Extraction. Approximately $20 \mathrm{~g}$ of $P$. ginseng that had been chopped into small pieces was weighed and placed in a $250-\mathrm{mL}$ glass bottle containing acetonitrile $(100 \mathrm{~mL})$, and then sodium chloride $(18 \mathrm{~g}$ ) was added to the mixture. The mixture was homogenized at $3000 \mathrm{rpm}$ for $5 \mathrm{~min}$ in a highspeed homogenizer (SMT High-Flex. HG-92. Japan). After the extraction procedure. the solution was transferred to a separation funnel and vigorously shaken for $3 \mathrm{~min}$ and the extract was centrifuged for $5 \mathrm{~min}$ at $3000 \mathrm{rpm}$. The organic layer was collected in a round-bottomed flask. and concentrated to dryness in a rotary evaporator (Büchi Rotavapor R114. Gennany) at $40^{\circ} \mathrm{C}$ in a Buchi Waterbath B-480.

Purification. The $\mathrm{NH}_{2}$ cartridge (Phenomenex. Torrance. Calif. USA) was washed with $6 \mathrm{~mL}$ dichloromethane (for activation). and the dried residue was dissolved in $2 \mathrm{~mL}$ of $n$-hexane. The mixture was then pre-loaded in the cartridge and eluted with $15 \mathrm{~mL}$ of dichloromethane containing $5 \%$ methanol. The elutent was filtered through sodium sulphate. evaporated at $40^{\circ} \mathrm{C}$, and dissolved in $2 \mathrm{~mL}$ of methanol: water $(1: 1, \mathrm{v} / \mathrm{v})$. The extract was then transferred to a $5 \mathrm{~mL}$ vial for chromatographic analysis. The details of the chromatographic conditions are shown in Table 2.

Analyte recovery rate. The extraction efficiency (recovery rate) of these pesticides was detenmined in triplicate at 2 fortification levels in spiked samples of blank $P$. ginseng (i.e. containing none of the pesticides of interest). The samples were processed according to the extraction procedure just described. The recovery rate was calculated by comparing peak areas for spiked ginseng (with known amounts of pesticides in the range of calibration curves concentrations) with standard solutions dissolved in organic solvent and injected directly into the analytical colunun.

\section{Results and Discussion}


Table 3. Determination coefficient, rate of recovery, RSD, LOD, LOQ and MRL tor the pesticides tested"

\begin{tabular}{|c|c|c|c|c|c|c|c|c|}
\hline No & Compound & $F^{*}$ & $\begin{array}{l}\text { Fortification levels } \\
\text { (ppm) }\end{array}$ & $\begin{array}{c}\text { Recovery rate } \\
(\%)\end{array}$ & $\begin{array}{l}\text { RSD } \\
(\%)\end{array}$ & $\begin{array}{l}\text { LOD } \\
\text { (ppm) }\end{array}$ & $\begin{array}{l}\mathrm{LOQ} \\
\text { (ppm) }\end{array}$ & $\begin{array}{l}\mathrm{MRL} \\
\text { (ppm) }\end{array}$ \\
\hline \multirow[t]{2}{*}{1} & Carbendazim & 0.9986 & 12.5 & $93.2 \pm 3.57$ & 3.8 & 0.039 & 0.12 & 0.125 \\
\hline & & & 1.6 & $118.8 \pm 10.50$ & 8.8 & & & \\
\hline \multirow[t]{2}{*}{2} & Cyazofamid & 0.9995 & 17.3 & $80.6 \pm 0.66$ & 0.8 & 0.054 & 0.16 & 0.075 \\
\hline & & & 2.2 & $80.2 \pm 5.13$ & 6.4 & & & \\
\hline \multirow[t]{2}{*}{3} & Diethofencarb & 0.9999 & 4.7 & $97.4 \pm 1.31$ & 1.4 & 0.015 & 0.05 & 0.125 \\
\hline & & & 0.6 & $81.2 \pm 0.00$ & 00 & & & \\
\hline \multirow[t]{2}{*}{4} & Pyrimethanil & 0.9999 & 3.1 & $72.7 \pm 0.67$ & 0.9 & 0.010 & 003 & 0.075 \\
\hline & & & 0.4 & $72.4 \pm 3.92$ & 5.4 & & & \\
\hline
\end{tabular}

"Pesticide components were analyzed by HPLC. $r$ ", determination coefficient: RSD. relative standard deviation: LOD. limit of determination: LOQ. limit of quantitation: MRL. maximum residue limit.
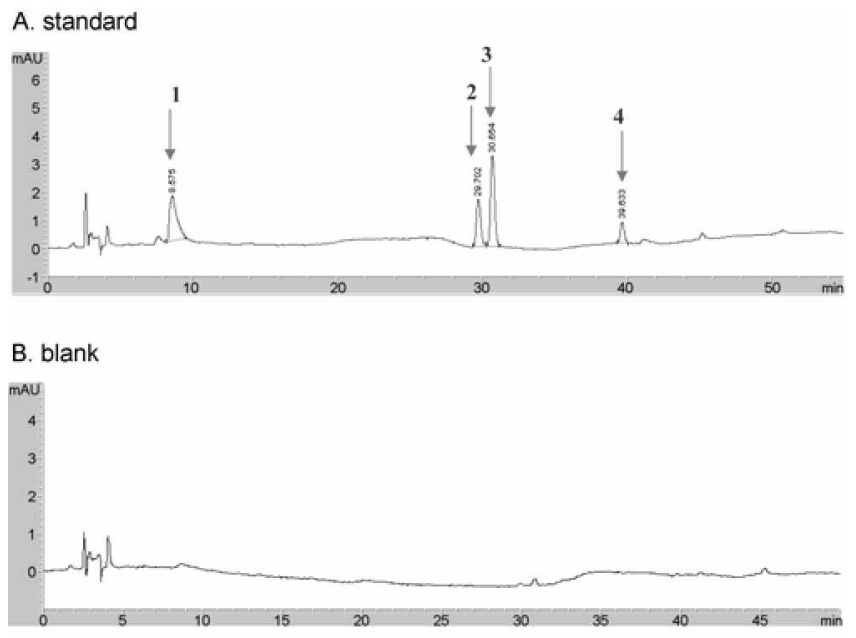

C. ginseng real samples

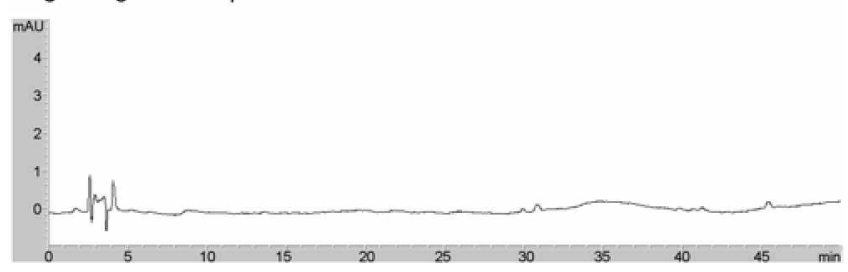

Figure 1. Typical HPLC-UVD chromatograms of carbendazim (1.6 ppm), cyazotamid (2.2 ppm), diethotencarb $(4.7 \mathrm{ppm})$ and pyrinethanil ( 3.1 ppmn) standards dissolved in organic solvent (A), blank (B), and ginseng (real) samples (C). See Table 1 for peak identification.

Linearity. The linearity of all the pesticides was determined using blank samples. Good determination coefficients were obtained for all of the compounds and ranged from 0.9986 to 0.9999 (Table 3)

Repeatability. The repeatability of our chromatographic findings was determined by analyzing a sample spiked at 50 $\mathrm{ppm}$. The sample was injected 10 times with an automatic injector. and the relative standard deviation (RSD) values for retention times ranged from $0.02 \%$ to $0.04 \%$ : whereas for peak areas the values ranged from $1.1 \%$ to $4.2 \%$. Therefore. the repeatability of the results of the HPLC analysis achieved by automatic injection was very good.

Stability. Stock standard solutions and working solutions were found to be stable for at least 3 months and 1 week. respectively, when stored at $4^{\circ} \mathrm{C}$. Moreover, when the stability of a fortified blank sample kept in the autosampler for 24 $\mathrm{h}$ was assayed. differences of $<3 \%$ were obtained.

Specificity. The specificity of the proposed procedure was assessed by analyzing blank samples. The absence of background peaks above a signal-to-noise ratio of 3 at the retention time for each pesticide indicated that no interference occurred (Figure 1).

Recovery. The rate of recovery for all pesticides ranged from $72.4 \%$ to $118.8 \%$. with RSDs lower than $9 \%$ (Table 3 ). These results show that the rates of recovery for pesticides were good. These values are similar to those reported elsewhere for other pesticide detected in ginseng. ${ }^{11-13}$

Detection and quantification limits. Both limits of detection (LOD) and limits of quantitation (LOQ) were calculated based on the minimum standard concentration detected by the analytical instrument as a peak. To determine this concentration. extracts of nonfortified ginseng (referred to as blanks in this study) were analyzed. Around each retention time of interest. signals below a certain response were considered noise. and the average noise. in terms of $\mathrm{Hz}$ or counts. was manually calculated. To qualify as a peak. a signal's response had to be either equal to (or higher than). three times the average noise in case of LOD or 10 times the average noise for LOQ. ${ }^{1+}$ Table 3 summarizes the LOD and LOQ for each pesticide. The LODs varied from 0.010 to $0.054 \mathrm{ppm}$. and the LOQs were lower than the maximum residue limits (MRLs) authorized by the Korean Food and Drug Administration. ${ }^{15}$ except for cyazofamid.

Analysis of ginseng samples. The analytical method was applied to the analysis of ginseng samples. No pesticide residue was found in any of the ginseng samples analyzed in this study (Figure 1).

\section{Conclusions}

We have described a method for monitoring the levels of certain pesticides in $P$. ginseng (C. A. Meyer) using HPLC. This method was simple. easy. and inexpensive and provided linear and repeatable data. For all the pesticides. the sensitivity of this method was good enough to ensure a reliable determination at pesticide levels that were much 
lower than the respective MRLs established by the Korean FDA. With slight modifications, it might also be applicable to other matrices.

\section{References}

1. Saito. H. Intemational Ginseng Seminar: Tokvo. 1989.

2. Saito. H.: Yoshida. Y: Takagi. K. Jpn. J. Pharmacol 1974. 24. 119

3. Duke, I. A. Ginseng: A Concise Hand Book: Reference Publication Ine.: Michigan, USA 1989.

4. Li, T. S. C.: Mazza, G.: Cottrell. A. C.: Gao, L. J. Agric. Food Chent. 1996. H.717

5. YokoZawa. T.: Seno. H.: Oura. H. Chem. Pham. Bull. Tokvo! 1975. 23. 3095 .

6. Takahashi. M.: Tokllyama. S.: Kaneto. H. Jpm. J. Pharmacol
$1992,59,399$

7. Kiml. Y. H.: Park. K. H.: Rho. H. M. J. Biol Chem. 1996. 271. 24539.

8. Zhang. D.: Yasuda. T.: Yu. Y.: Zheng. P.: Kawabata. T.: Ma. Y.: Okada, S. Free Radic. Biol Med 1996, $20,145$.

9. Xiaoguang. C.: Hongyan. L.: Xiaohong. L.: Zhaodi. F: Yan, L.; Lihua. T.: Rui. H. J. Ethophamacol $1998,60.71$.

10. Ohh. S. Korem J. Ginseng Sci, 1986. 5.73.

11. Zhao. Y.: Chen. J.: Wang. X. Wei Sheng Iam Jiu 1999. 28.53.

12. Sohn1. S. H.: Kim. S. K.: Kang. H. G.: Wee. T. J. J. Chomatogr .A 2004. 1042, 163.

13. Park, Y. S; Abd El-Aty. A. M.; Choi, J. H.; Cho, S. K.: Shin. D $\mathrm{H}$ : Shim. J. H. Bioned Chronatogr: 2007, 21.29

14. Adou. K.: Bontoyat1. W. R.: Sweeney. P. I. J. Agric. Food Chen 2001. +9.4153

15. Korea Food and Drug Administration: MRLs for Pesticides in Foods, Seoul. Korea, 2005. 